\title{
Exophytic Cerebellar Glioblastoma in the Cerebellopontine Angle: Case Report and Review of the Literature
}

\author{
Masahide Matsuda $^{1}$ Kuniyuki Onuma ${ }^{1}$ Kaishi Satomi ${ }^{2}$ Kei Nakai ${ }^{1}$ Tetsuya Yamamoto ${ }^{1}$ \\ Akira Matsumura ${ }^{1}$
}

${ }^{1}$ Department of Neurosurgery, Faculty of Medicine, University of Tsukuba, Tsukuba, Ibaraki, Japan

2 Department of Diagnostic Pathology, Faculty of Medicine, University of Tsukuba, Tsukuba, Ibaraki, Japan

Address for correspondence Masahide Matsuda, MD, PhD, Department of Neurosurgery, Faculty of Medicine, University of Tsukuba, Tsukuba, Ibaraki 305-8575, Japan (e-mail: m-matsuda@md.tsukuba.ac.jp).

J Neurol Surg Rep 2014;75:e67-e72.

\begin{abstract}
Keywords

- glioblastoma

- exophytic

- cerebellopontine angle

- single-photon emission computed tomography

We report the unique case of a 69-year-old man with a cerebellar glioblastoma showing an exophytic growth pattern. Magnetic resonance imaging revealed a heterogeneously gadolinium-enhancing tumor in the right cerebellopontine angle. The preoperative differential diagnoses included an intraaxial tumor, such as high-grade glioma, and an extraaxial tumor, such as a meningioma or neurinoma. The tumor with a clear boundary was subtotally removed, except for the adhesion site to the petrosal vein, and the histologic diagnosis was glioblastoma. Immunohistochemical analyses demonstrated that the tumor cells were immunopositive for epidermal growth factor receptor and immunonegative for $\mathrm{p} 53$ mutation and IDH1 R132H, indicating that it had different genetic features from a typical cerebellar glioblastoma. Conventional radiotherapy with 60 Gy concurrent with temozolomide was performed, and the condition of the patient has remained stable for 24 months since the operation. Exophytic cerebellar glioblastoma should be considered in the differential diagnosis of cerebellopontine angle tumor.
\end{abstract}

\section{Introduction}

Glioblastoma, the most malignant and most common primary brain tumor in adults, is commonly located in cerebral hemispheres. Cerebellar glioblastoma is rare, with a frequency of 0 to $3.4 \%$, and it usually grows invasively into the surrounding normal tissue in the cerebellar hemisphere or the cerebellar vermis. ${ }^{1-3}$ The exophytic growth pattern of cerebellar glioblastoma is extremely rare. To date, only three cases of glioblastoma arising from the cerebellum with exophytic extension have been described in the English literature. ${ }^{4-6}$ We report a case of glioblastoma arising from the surface of the cerebellar hemisphere with exophytic extension into the cerebellopontine angle, mimicking an extraaxial tumor, and discuss the histopathologic and radiologic aspects.

received

October 7, 2013

accepted

November 15, 2013

published online

March 12, 2014 10.1055/s-0033-1364167. ISSN 2193-6358.

\section{Case Report}

A 69-year-old man without notable previous medical history presented to a local outpatient clinic with right facial pain. He had two episodes of pain lasting for an hour at a time. He was referred to our hospital because magnetic resonance (MR) imaging revealed a gadolinium (Gd)-enhancing mass in the right cerebellopontine angle. On admission, neurologic examination showed no deficit. Cranial nerve palsy, cerebellar ataxia, nystagmus, and pyramidal tract sign were not observed. The laboratory examination findings were essentially normal. MR imaging performed in our hospital showed a heterogeneously Gd-enhancing tumor in the right cerebellopontine angle without extension into the internal acoustic canal (-Fig. 1). T2-weighted fast-field echo imaging revealed a low-intensity area within the mass, suggesting intratumoral

\footnotetext{
(c) 2014 Georg Thieme Verlag KG, Stuttgart · New York
}

License terms

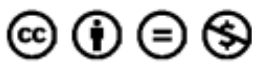



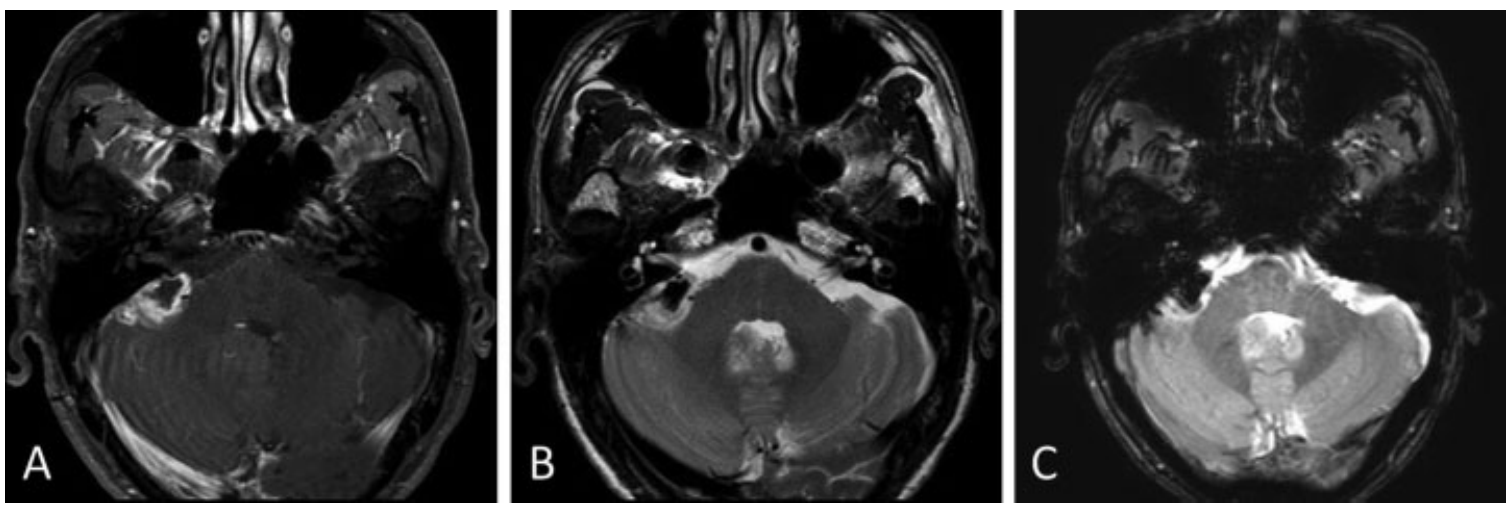

Fig. 1 Magnetic resonance imaging revealing a heterogeneously enhancing tumor with intratumoral hemorrhage in the right cerebellopontine angle. (A) Axial T1-weighted image with gadolinium. (B) Axial T2-weighted image. (C) Axial T2-weighted fast-field echo imaging.

hemorrhage. Of note, there was no peritumoral edema. ${ }^{201}$ Thallium (Tl) single-photon emission computed tomography (SPECT) performed 15 minutes (early) and 4 hours (delayed) after the intravenous injection of ${ }^{201} \mathrm{Tl}$ chloride $74 \mathrm{MBq}$ showed homogeneous hot tumor uptake on both early and delayed images (-Fig. 2). The calculated $\mathrm{Tl}$ index for the tumor and normal tissue in early and delayed images was 2.012 and 1.810 , respectively. The retention index, calculated as the ratio of delayed to early $\mathrm{Tl}$ index, was 0.90 , suggesting the malignant nature of the tumor. The preoperative differential diagnoses included an intraaxial tumor, such as high-grade glioma, and an extraaxial tumor, such as meningioma or vestibular schwannoma. He underwent a right retrosigmoid craniotomy in the lateral position. An exophytic reddish hypervascular tumor was identified on the surface of the lateral cerebellar hemisphere. Although the petrosal vein was completely encased by the tumor, the seventh and eighth cranial nerves were not encased but adjacent to the tumor. The boundary between the cerebellar hemisphere and the tumor margin was substantially clear. Except for the adhesion site to the petrosal vein, the tumor was subtotally removed.
The postoperative course was uneventful. Postoperative MR imaging revealed a faint residual tumor adjacent to the petrosal vein and no complications related to surgery (-Fig. 3). Histopathologic examination revealed a highly cellular tumor composed predominantly of spindle-shaped or multipolar astrocytic cells with mild nuclear hyperchromasia, eosinophilic cytoplasm, and frequent mitotic figures (-Fig. 4). Microvascular proliferation with a swollen endothelium was a predominant feature; however, there was no appearance of necrosis. Histopathologic analysis identified the tumor as glioblastoma. Immunohistochemical staining of the tumor cells revealed diffuse glial fibrillary acidic protein activity with a proliferation rate of $22.8 \%$, as determined by the MIB- 1 labeling index. None of the tumor cells were p53 mutation positive. Epidermal growth factor receptor (EGFR) cytoplasmic immunoreactivity was observed. No immunoreactivity was detected for a monoclonal antibody specific for isocitrate dehydrogenase (IDH) $1 \mathrm{R} 132 \mathrm{H}$ mutation. ${ }^{7}$ Conventional radiotherapy with $60 \mathrm{~Gy}$ concurrent with temozolomide was performed. Additionally, adjuvant temozolomide was continued based on the Stupp regimen. ${ }^{8}$ Although MR imaging 13 months
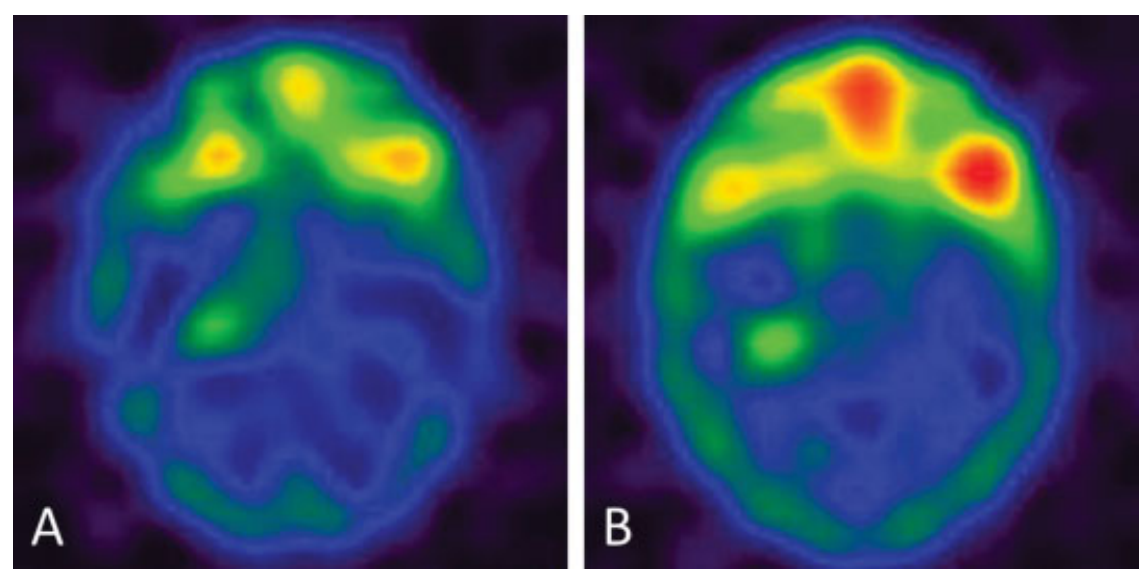

Fig. 2 Thallium TI 201 single-photon emission computed tomography showing homogeneous hot tumor uptake on both (A) early and (B) delayed images. 

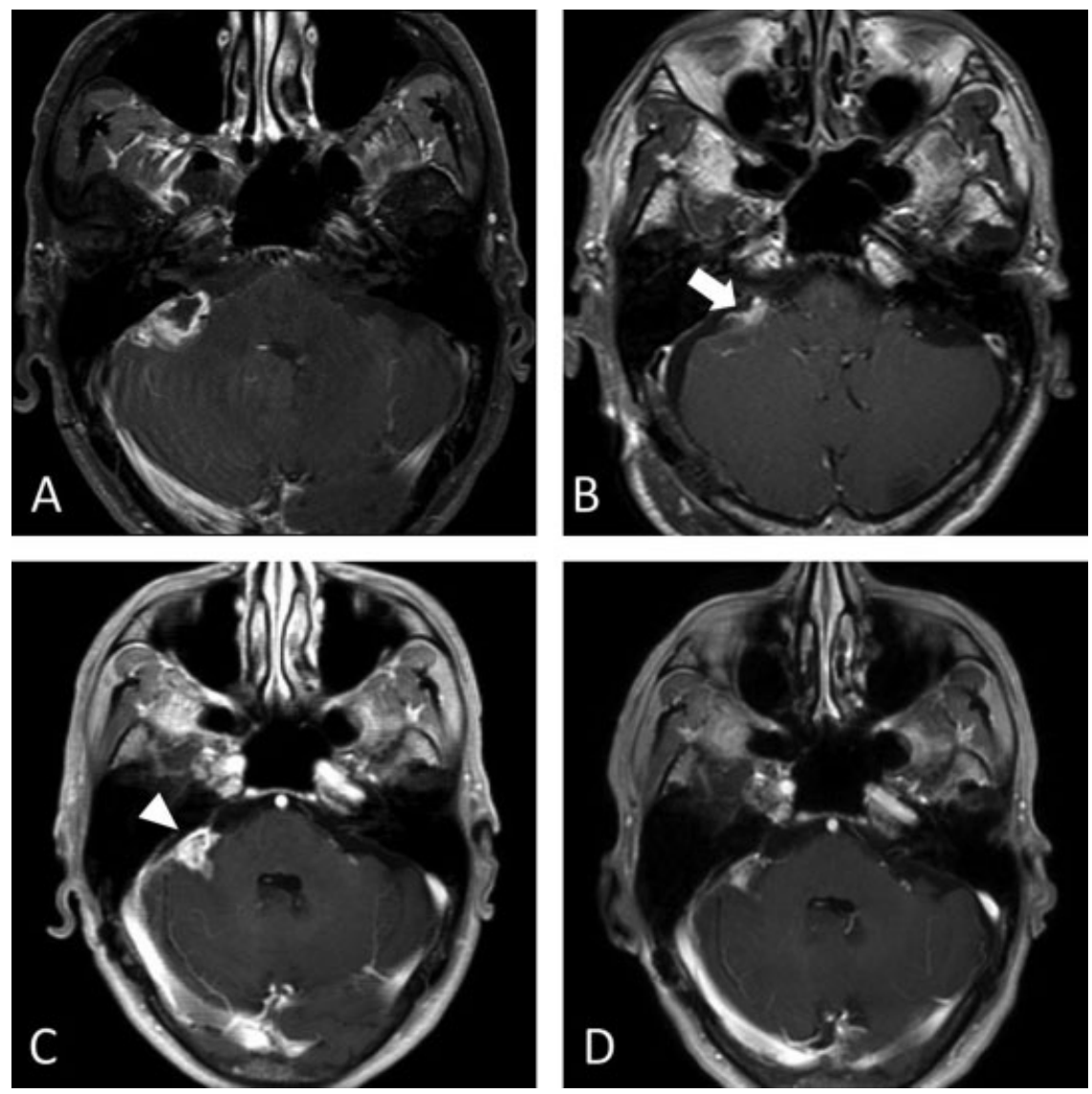

Fig. 3 Axial T1-weighted image with gadolinium (A) before the operation, (B) 1 day after the operation, (C) 13 months after the operation, and (D) 20 months after the operation. The arrow indicates the faint residual tumor adjacent to the petrosal vein. The arrowhead indicates enlargement of the residual enhancing mass.

after the operation suggested progression of the residual tumor, the mass size has remained unchanged for 7 months since then (-Fig. 3). Moreover, he has remained symptom free for 24 months since the operation.

\section{Discussion}

Cerebellar glioblastoma presenting with an exophytic growth pattern is an extremely rare entity. To our knowledge, only three cases have been reported in the literature (-Table $\mathbf{1}$ ). Yamamoto et al reported a case of glioblastoma arising from the cerebellar hemisphere with exophytic extension into the crural and quadrigeminal cistern. ${ }^{6}$ The patient underwent subtotal removal of the tumor followed by radiotherapy with concomitant chemotherapy. The patient died 1 year after the operation due to regrowth of the tumor. Kasliwal et al reported a pediatric case of multicentric glioblastoma including a cerebellar lesion with exophytic extension into the cerebellopontine angle cistern. ${ }^{4}$ The patient underwent biopsy of the cerebellopontine angle lesion. The patient died 2 months after the operation due to complicated aspiration pneumonia. Linsenmann et al reported a case of glioblastoma arising from the cerebellar tonsil with exophytic extension into the cisterna magna. ${ }^{5}$ The patient underwent gross total removal of the tumor followed by radiotherapy with concomitant temozolomide chemotherapy. Histopathologic examination of these three cases revealed typical features of glioblastoma such as cellular pleomorphism, microvascular proliferation, and focal necrosis. The detailed immunohistochemical features were insufficiently described in these reports.

It was reported that cerebellar glioblastomas typically exhibit p53 mutation immunopositivity and EGFR immunonegativity, suggesting a genetic feature of secondary glioblastoma. ${ }^{9-11}$ In contrast, immunohistochemical analyses of the present case demonstrated that the tumor cells were immunopositive for EGFR and immunonegative for p53 mutation and IDH1 R132H. The present case seems to have different genetic features from typical cerebellar glioblastoma based on immunohistochemistry. Utsuki et al reported immunohistochemical studies of IDH1 mutations in cerebellar glioblastomas. ${ }^{11}$ Cerebellar glioblastoma was immunonegative for IDH1 mutations, implying a difference from secondary supratentorial glioblastoma, which tends to be immunopositive for IDH1 mutation. The present case was consistent with typical cerebellar glioblastoma in relation to 


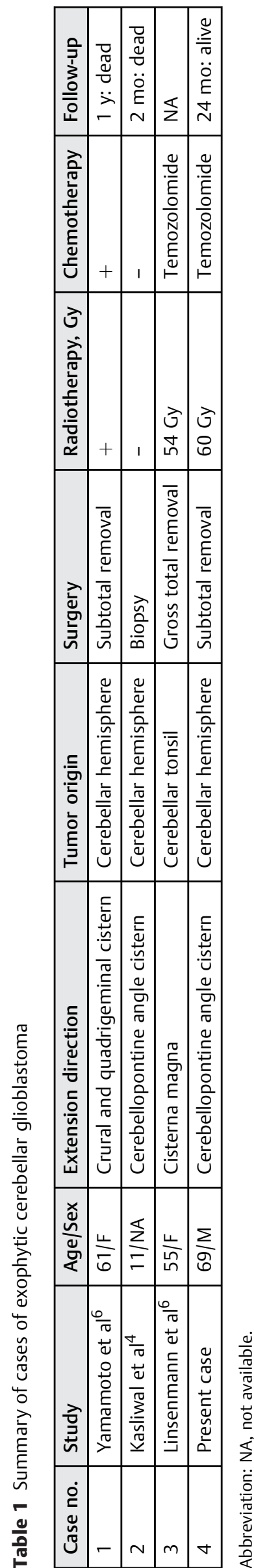

the observation of IDH1 mutations. Typical cerebellar glioblastoma generally grows locally and invasively into the surrounding normal tissue, occasionally into the brainstem or adjacent leptomeninges. ${ }^{6,12}$ The exophytic growth pattern observed in the present case might be attributed to the particular genetic alteration revealed by the immunohistochemical features.

Glioblastoma located in the cerebellopontine angle arises from various origins. Several authors have previously documented cases of exophytic pontine glioblastoma in the cerebellopontine angle. ${ }^{13-15}$ Cases of primary glioblastoma in the cerebellopontine angle arising from the proximal portion of the eighth cranial nerve have also been documented. ${ }^{16} \mathrm{~A}$ common problem among those cases, including the present case, is the difficulty of preoperative diagnosis. Glioblastoma in the cerebellopontine angle could be readily misdiagnosed as an extraaxial tumor such as a vestibular schwannoma or meningioma that occurs more commonly. Kuroiwa et al documented the MR features of posterior fossa glioblastoma, such as heterogeneous signal intensity (presence of hemorrhage), prominent heterogeneous and ring-like enhancement (necrosis) with poorly defined margins, and multicentricity. $^{2}$ Zito et al reported the radiologic features of posterior fossa glioblastoma, such as lack of peritumoral edema and little mass effect. ${ }^{17}$ However, there are no definite MR features available to differentiate glioblastoma in the cerebellopontine angle from the other benign extraaxial tumors. Several reports have stated the utility of TI SPECT, which can provide useful information about the nature of tumor in the differential diagnosis between malignant and benign brain tumors. ${ }^{18,19}$ In particular, the retention index, defined as the ratio of delayed to early Tl uptake, has been demonstrated to be useful in differential diagnosis. In those reports, the retention index of malignant tumors was significantly higher than that of benign tumors, including meningioma and schwannoma, and the threshold value was 0.65 to $0.70 .^{18,19}$ In the present case, the retention index of cerebellar glioblastoma in the cerebellopontine angle was 0.90 , which is consistent with supratentorial glioblastoma in previous reports. Accordingly, the retention index of TI SPECT could make it possible to differentiate high-grade glioma in the cerebellopontine angle from the other extraaxial tumors preoperatively; thus it could help to design an appropriate strategy of operation.

Although defined standard therapy has been established for supratentorial glioblastoma, the optimal treatment for cerebellar glioblastoma is yet to be defined because of its rarity. Several reports have supported the application of chemoradiotherapy based on the Stupp regimen to cerebellar glioblastoma. ${ }^{1,20}$ In the present case, radiotherapy with temozolomide was applied to cerebellar glioblastoma with an exophytic growth pattern, and a relatively good clinical course was achieved. Thus radiotherapy with temozolomide may also be effective for exophytic cerebellar glioblastoma, which seems to be genetically different from typical cerebellar glioblastoma. 

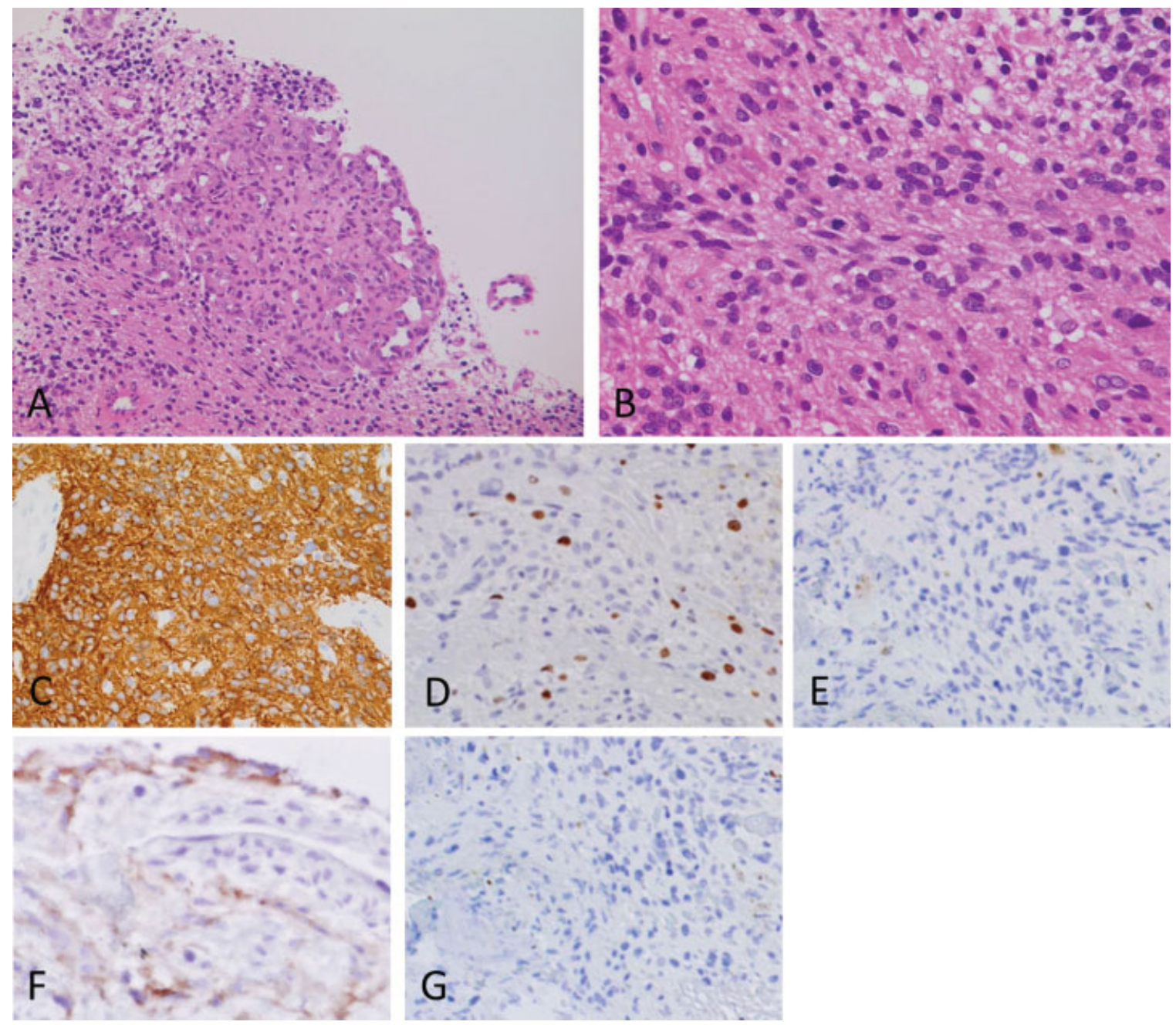

Fig. 4 (A) Photomicrograph showing a highly cellular tumor composed predominantly of spindle-shaped or multipolar astrocytic cells.

Microvascular proliferation with swollen endothelium was seen in the tumor; however, there was no appearance of necrosis. (B) Photomicrograph showing cells with mild nuclear hyperchromasia, eosinophilic cytoplasm, and frequent mitotic figures. (C) Immunohistochemical analysis showing diffuse staining for glial fibrillary acidic protein. (D) MIB-1 labeling index $22.8 \%$. (E) Immunohistochemical analysis showing negative staining for p53 mutation. (F) Immunohistochemical analysis showing positive staining for epidermal growth factor receptor. (G) Immunohistochemical analysis showing negative staining for a monoclonal antibody specific for IDH1 R132H mutation. (A, B) Hematoxylin and eosin staining; (A) magnification $\times 100$. $(B-G)$ magnification $\times 400$.

\section{Conclusions}

Exophytic cerebellar glioblastoma should be considered in the differential diagnosis of a cerebellopontine angle mass with atypical radiologic features such as heterogeneous signal intensity and poorly defined margins, although it is an extremely rare occurrence. Tl SPECT would be useful in achieving an accurate preoperative diagnosis for atypical cerebellopontine angle tumor, which leads to an optimal strategy for surgery.

\section{Acknowledgments}

We are very grateful to M. Shibuya of the Department of Diagnostic Pathology, Tokyo Medical University Ibaraki Medical Center, Ibaraki, for his comments on the pathologic findings.

\section{References}

1 Grahovac G, Tomac D, Lambasa S, Zoric A, Habek M. Cerebellar glioblastomas: pathophysiology, clinical presentation and management. Acta Neurochir (Wien) 2009;151(6):653-657

2 Kuroiwa T, Numaguchi Y, Rothman MI, et al. Posterior fossa glioblastoma multiforme: MR findings. AJNR Am J Neuroradiol 1995;16(3):583-589

3 Stark AM, Nabavi A, Mehdorn HM, Blömer U. Glioblastoma multiforme-report of 267 cases treated at a single institution. Surg Neurol 2005;63(2):162-169; discussion 169

4 Kasliwal MK, Gupta DK, Mahapatra AK, Sharma MC. Multicentric cerebellopontine angle glioblastoma multiforme. Pediatr Neurosurg 2008;44(3):224-228

5 Linsenmann T, Monoranu CM, Westermaier T, Varallyay C, Ernestus RI, Vince GH. Exophytic glioblastoma arising from the cerebellum: case report and critical review of the literature. J Neurol Surg A Cent Eur Neurosurg 2013;74(4):262-264

6 Yamamoto M, Fukushima T, Sakamoto S, et al. Cerebellar gliomas with exophytic growth-three case reports. Neurol Med Chir (Tokyo) 1997;37(5):411-415 
7 Kato Y, Jin G, Kuan CT, McLendon RE, Yan H, Bigner DD. A monoclonal antibody IMab-1 specifically recognizes IDH1R132H, the most common glioma-derived mutation. Biochem Biophys Res Commun 2009;390(3):547-551

8 Stupp R, Mason WP, van den Bent MJ, et al; European Organisation for Research and Treatment of Cancer Brain Tumor and Radiotherapy Groups; National Cancer Institute of Canada Clinical Trials Group. Radiotherapy plus concomitant and adjuvant temozolomide for glioblastoma. N Engl J Med 2005;352(10):987-996

9 Ohgaki H, Kleihues P. Genetic pathways to primary and secondary glioblastoma. Am J Pathol 2007;170(5):1445-1453

10 Saito T, Hama S, Kajiwara Y, et al. Prognosis of cerebellar glioblastomas: correlation between prognosis and immunoreactivity for epidermal growth factor receptor compared with supratentorial glioblastomas. Anticancer Res 2006;26(2B):1351-1357

11 Utsuki S, Oka H, Miyajima Y, Kijima C, Yasui Y, Fujii K. Adult cerebellar glioblastoma cases have different characteristics from supratentorial glioblastoma. Brain Tumor Pathol 2012;29(2): 87-95

12 Kopelson G. Cerebellar glioblastoma. Cancer 1982;50(2):308-311
13 Rasalingam K, Abdullah JM, Idris Z, et al. A rare case of paediatric pontine glioblastoma presenting as a cerebellopontine angle otogenic abscess. Malays J Med Sci 2008;15(1):44-48

14 Salunke P, Sura S, Tewari MK, Gupta K, Khandelwal NK. An exophytic brain stem glioblastoma in an elderly presenting as a cerebellopontine angle syndrome. Br J Neurosurg 2012;26(1):96-98

15 Swaroop GR, Whittle IR. Exophytic pontine glioblastoma mimicking acoustic neuroma. J Neurosurg Sci 1997;41(4):409-411

16 Wu B, Liu W, Zhu H, Feng H, Liu J. Primary glioblastoma of the cerebellopontine angle in adults. J Neurosurg 2011;114(5):1288-1293

17 Zito JL, Siva A, Smith TW, Leeds M, Davidson R. Glioblastoma of the cerebellum. Computed tomographic and pathologic considerations. Surg Neurol 1983;19(4):373-378

18 Otsuka $\mathrm{H}$, Shinbata $\mathrm{H}$, Hieda $\mathrm{M}$, et al. The retention indices of 201Tl-SPECT in brain tumors. Ann Nucl Med 2002;16(7):455-459

19 Taki S, Kakuda K, Kakuma K, et al. 201Tl SPET in the differential diagnosis of brain tumours. Nucl Med Commun 1999;20(7):637-645

20 Tsung AJ, Prabhu SS, Lei X, Chern JJ, Benjamin Bekele N, Shonka NA. Cerebellar glioblastoma: a retrospective review of 21 patients at a single institution. J Neurooncol 2011;105(3):555-562 